\title{
Influence of sensitivity and specifity of measuring methods on their informativity and hardware requirements
}

\author{
N K Andreev* \\ Department of Measurement Instruments and Mechatronics, Kazan State Power Engineering University, 51 Krasnosel'skaya Street, \\ Kazan, 420066, Russia
}

\begin{abstract}
This paper is devoted to discussion how the sensitivity and specificity of a physical quantity under measurement to other physical, chemical and biologic features influence the information volume circulating in a smart system that, i.e. to the degree of smartness. Measurement systems in the fields of magnetic resonance imaging (MRI), acoustic inspection and optical control of high voltage insulators are discussed in connection with the devices development. It is considered how the addition of new measuring channels in optics, acoustics and MRI influences the images' quality and how it affects data processing and hardware

requirements.
\end{abstract}

\section{Introduction}

Here we define Smart Systems as devices that incorporate functions of sensing, actuation and control. They can be capable of describing and analyzing a situation, and taking decisions based on the available data in a predictive or adaptive manner, thereby performing smart actions.

This paper is devoted to discussion how the sensitivity and specificity of a physical quantity under measurement to other physical, chemical and biologic features influence the information volume circulating in a smart system, i.e. to the degree of smartness. The greater is the amount of information about the object under study, the more opportunities for making management decisions there are. This is especially important in the fields of scientific research and medical diagnosis.

The discussion concerns measurement systems in the fields of magnetic resonance imaging (MRI), acoustic inspection, optical control of high voltage insulators in connection to the work for development of devices. Hardware requirements will be discussed later after considering the basics of NMR imaging.

\section{Methods}

\subsection{The sensitivity and specifity}

The sensitivity is an important factor from several points of view. The sensitivity is a smallest change in a measured physical quantity that an instrument is capable of detecting [1]. In terms of sensor selection, sensitivity to a specific physical phenomenon is important. The usual definition of sensitivity is as follows: for a quantity
$Q$ which is a function of other quantities $M(B), t, T, f(T)$, $T, \omega:$

$$
Q=Q[M(B), t, T, f(T), \omega]
$$

where, for example, $M$ is the magnetization, $B$ is the magnetic field induction, $t$ is time, $T$ is temperature, $f$ is humidity, $\omega$ is frequency. A small change of $Q$ can be represented as

$$
\begin{aligned}
& \Delta Q=\frac{d Q}{d M} \frac{d M}{d B} \Delta B+\frac{d Q}{d t} \Delta t+ \\
& +\frac{d Q}{d T} \Delta T+\frac{d Q}{d f} \frac{d f}{d T} \Delta T+\frac{d Q}{d \omega} \Delta \omega
\end{aligned}
$$

Here derivatives $\frac{d Q}{d M}, \frac{d Q}{d t}, \frac{d Q}{d T}, \frac{d Q}{d f}, \frac{d Q}{d \omega}$ are sensivities of the quantity $Q$ to small changes of its corresponding arguments $, t, T, f, \omega$. But these arguments can be sensitive to variations of other physical, chemical or biological quantities. Some of these sensitivities are expressed through other derivatives which, in turn, can indicate the presence of the "specifity" of the quantity under measurement to small changes of intrinsic features of the object of interest. In this way, it is possible to construct a feature space that most accurately reflects the properties of the object under study, in order to subsequently make an adequate decision on its assignment to a certain type.

To be smart, the methods of measurement should be sufficiently sensitive and informative. The sensitivity allows one to distinguish signal from noise if the signal amplitude is several times higher than the noise

* Corresponding author: ngeikandreev@ gmail.com 
amplitude $(N \geq 2-3)$. But for imaging systems, high contrast sensitivity $\left(\frac{C}{N}\right)$ is also important [1].

The contrast enhancement is associated with a change of $\left(\frac{d M}{d X}\right) \Delta X$, where $M$ is magnetization, $\Delta X$ is the difference between the values of parameter $X$ in the adjacent areas 1 and 2 of the image, i.e. $C(12)=$ $\Delta X(1)-\Delta X(2)$. Therefore, it is necessary to propose pulse sequences to control the dynamics of a nuclear magnetization with high sensitivity to the inspected parameter. This means that, if the parameters $\Delta X$ themselves depend on other parameters $Y: \Delta X=$ $\frac{d X}{d Y} \Delta Y$, the researcher should apply such pulse sequence and create such a quantity $Y$ which will give the greatest change in contrast $[2-5]$.

\subsection{Methods to enhance sensitivity}

In magnetic resonance imaging, the sensitivity is proportional to the magnetic field strength [6]. The direct method to enhance sensitivity is to enhance the magnetic field strength $B_{0}$. The nuclear magnetic moment can be enhanced through the magnetization transfer from the electron with a high magnetic moment to the nuclear magnetic moment by using dynamic nuclear polarization and chemically induced dynamic nuclear polarization effects [7]. On the contrary, in the field of acoustic measurements, the sonic waves are attenuated when propagate in solids. Therefore, sometimes, usage of low frequencies is more beneficial $[8,9]$.

Other ways of sensitivity enhancement are signal filtration, signal averaging, multisensor signal recording and correlation reception [1].

It is well known that the simultaneous multi-channel signal recording is, in some way, equivalent to the signal averaging and increasing the signal/noise ratio, but for the shorter time [1]. However simultaneous signal recording at various frequencies or using sensors of different nature is the accumulation of information about different features. If these different features can be used together, one can get additional gain in sensitivity. For example, such gain in sensitivity was obtained by using three channel recording of images of high voltage insulators in infrared, visible and ultraviolet ranges with subsequent simultaneous image processing [10]. The defects, that were not visible after recording images by the infrared channel, became clearly visible after conscientious processing of three images taken in different channels, i.e. the contrast/noise ratio was essentially enhanced.

On the other hand, the application of a new additional signal recording channel provides new information. For example, during the inspection of oil wells $[8,9]$ by acoustic sounding, in addition to recording the time dependence of echo signals, we began to study the change (decreasing) in attenuation of the signal envelope depending on the duration of the well operation, and the signs of gradual destruction of the cement shell were revealed.

The method of multi-frequency acoustic sounding allowed us to examine both near and remote areas of oil wells: for near areas high frequency waves were used, and for remote areas lower attenuating low frequency waves were used $[8,9]$.

\subsection{Main aspects of NMR imaging}

\subsubsection{Nuclear magnetic resonance (NMR)}

For visualization of biological tissues, NMR as a physical phenomenon is a very good choice. The effect of NMR is sensitive to the presence of magnetic nuclei, for example, hydrogen nuclei ${ }^{1} \mathrm{H}$ in water $[2,4]$. Hydrogen nuclei are part of water molecules, which are abundant in biological objects. The object of study is placed in a magnetic field $B_{0}$. A hydrogen nuclear ${ }^{1} \mathrm{H}$ has a magnetic moment:

$$
\mathbf{M}_{0}=N \frac{\gamma^{2} h^{2} I(I+1)}{3 k T} \mathbf{B}_{0} .
$$

In the magnetic field, hydrogen nuclear moments are oriented mainly along the magnetic field $B_{0}$. At the same time they precess. The precession frequency is proportional to the magnitude of the external magnetic field $B$ :

$$
\omega=\gamma B
$$

\subsubsection{Magnetic resonance imaging (MRI)}

In MRI pulse methods of excitation are used. They provide more information per a unit time than frequency scanning methods [3-5].

The object under study is excited by pulses of alternating magnetic field at a precession frequency - at the resonance frequency. Water, depending on its structure and state, has different molecular and macroscopic mobility. This fact leads to that two time characteristics of water, $T_{1}$ and $T_{2}$, are sensitive of a sample structure under study. These characteristic constants can be measured by varying the pulse duration and the time delay between the pulses $[2,11]$.

The superposition of gradients of the magnetic field along the axes $X, Y, Z$

$$
G_{x}=\frac{\partial B}{\partial X}, G_{y}=\frac{\partial B}{\partial Y}, G_{z}=\frac{\partial B}{\partial Z}
$$

on the sample under study makes it possible to change the resonance frequency of individual sections of the object. A resonant excitation by narrow-band pulses of radio-frequency magnetic field makes it possible to 
record the signal from these parts of a sample, that is, to produce magnetic coding of their spatial position [6].

Pulse sequences contain combinations of selective radiofrequency pulses and pulses of magnetic field gradients for the slice selection, and, following them, NMR signal phase coding gradients.

\subsubsection{NMR for motion measurement}

NMR is sensitive to the movement of resonant nuclei and other nuclei that magnetically interact with them. It is customary to distinguish two types of molecular motion: diffusion and flow. Now researchers have developed many pulse sequences to measure flow and diffusion velocities $v, D$, as well as flow and diffusion structures [4-6] using pulses of magnetic field gradients $G_{i}$.

For the conditions of pulse NMR experiments, the effect of laminar flow in pipes without magnetic field gradients can be detected as an attenuation of echo signal amplitudes because the part of the spins excited by a first radiofrequency pulse is removed from the volume of a RF coil for the moments of echo maxima.

In the presence of magnetic field gradients laminar flow in pipes gives the phase shift of magnetization. Therefore, laminar flow results in a $\operatorname{sinc} X=\frac{\sin X}{X}$ behavior and the doubled frequency of oscillation of the echoes compared to a cosine behavior and oscillations for plug flow. But the spin echo changes its form near the zeroes of the function $\operatorname{sinc} X$. It obtains an additional minimum at the center and has four peaks in this case.

Flow motions in closed volumes can be stimulated by gradients of potentials of any physical or chemical nature. Here, for example, the thermal convection and acoustic-pumping driven convections can be considered. Frequently, the thermal or forced convection generates periodical structures in a fluid consisting of cells with a circulating liquid. It was shown in $[11,12]$ that acoustic pressure in a closed volume can produce two symmetrical circular streams flowing in opposite directions at frequencies $\pm \Delta \omega$. These circular streams cause the kinematic frequency shifts from resonance at $\omega_{0}$ and, consequently, produce corresponding time shifts $\pm \Delta \mathrm{t}$ of the echo peaks from the central position at $t=2 \tau$ (splitted echo signals). Thus, it has been proved $[13,14]$ that the splitted (two-maxima) echo signals can be observed without magnetic field gradients for such cases.

\subsubsection{Functional magnetic resonance measurement (fMRI)}

Ogawa, Lee, and co-workers first demonstrated at high field (7 and $8.4 \mathrm{~T}$ ) and high resolution (65/xm pixels) that changes in paramagnetic deoxyhemoglobin $(\mathrm{dHb})$ levels can be correlated with local blood flow and local brain activity [15]. A paramagnetic ion has the greater magnetic moment than surrounding diamagnetic molecules and changes magnetic fields at positions of these molecules. Functional MR imaging (fMRI) based upon the Blood Oxygen Level Dependent (BOLD) effect is a new tool for understanding brain function and specifically allowing the correlation of physiological activity with anatomical location [16].

\section{Results and discussion}

It is usually assumed that Smart Systems consist of sensors for signal acquisition, command and control units that take decisions and give instructions based on the available information, elements transmitting the information to the command and control unit, components transmitting decisions and instructions, actuators that perform or trigger the required action.

\subsection{Hardware and software in one-sided well- logging}

Consider an example of the structure of the device for acoustic well-logging $[9,10]$. The characteristic property of the proposed well-logging method is that longitudinal waves at $1-20 \mathrm{kHz}$ are used for inspection and only single-sided access is needed for transmitting and receiving transducers located at a head of a casing. The method reduces to the pulse exciting of longitudinal elastic waves in a body of a pipe coated by cement and receiving of echo signals from defects of bonding of a cement slice to the surfaces of pipes. The time of flight of a wave from the defect to transmitter (receiver) can be converted through the velocity of propagation of sonic waves in a steel pipe to the value of the correspondent source-defect distance.

Sonic waves moving in a pipe are attenuated. Amplitudes of detected signals are often very small and comparable with the amplitudes of various types of noise.

The detection, filtration and selection of the useful fraction of the signal are a complex problem. The corresponding computer programms are developed using the National Instruments LabVIEW environment. As a result, all operations concening control and synchronization of the apparatus under consideration, signal detection, registration, data processing and displaying are performed automatically under the computer control.

The carrier code is generated by the computer. Then a DAC unit converts a carrier code to an analog form which is amplified in turn by the power amplifier. An ADC unit connected by an interface to a receiver transducer serves for conversion of received echo signals to a digital form and control of the output level of the power amplifier.

The pulser used is an electronic device that can produce electrical pulses up to $3 \mathrm{kV}$ of amplitude. Driven by the pulser, the transmitter transducer connected to a power amplifier through the electric transformer generates sonic energy at desired frequencies in the range of 1-20 kHz. The length of the pulse can be changed from two to ten cycles of a carrier. The acoustic contact between transducers and the surface of the head of a pipe is provided through the slice of alabaster (gypsum) or other contact liquid. 
Single signal processing algorithm consists of successive stages of extraction of useful signal from noise, exponential normalization and defect calculation. The defect detection criterion is that the signal amplitude exceeds the threshold level. Comparison of the two signals can help to clarify the localization of defects obtained by sensing at different frequencies. It is possible to determine the trend towards new defects in periodic measurements during the life cycle of the well. The program calculates the relative area occupied by defective sections of the well and makes recommendations for further operation of the well on the basis of the results of diagnostics of its technical condition.

\subsection{Hardware and software in FMRI}

Even the first samples of MRI systems were constructed as intellectual measuring instruments [4-6]. We do not consider the features of hardware and software of the first-generation MRI devices here, but investigate these issues in devices of a functional MR-tomography at once as representatives of the most complex modern systems.

Typical fMRI studies are carried out with a $T 2 *_{-}$ weighted echo planar imaging (EPI) sequence acquiring images at a rate of 10 images/second for about 5-10 minutes $[15,16]$. This requires gradient rise times of about $<300 / n s$ for $25 \mathrm{mT} / \mathrm{m}$. A computer system capable of acquiring, reconstructing, and storing 3600-18 000 images per fMRI study is necessary. A typical hospital network with an effective throughput of $1 \mathrm{Mbit} / \mathrm{s}$ will transfer these images more than an hour. Since strong $\mathrm{T} 2 *$ weighting is required, the center of space should be acquired at about 40 -to $60 \mathrm{~ms}$ (at $1.5 \mathrm{~T}$ ). This, in turn, requires a very good shim for a sufficient image signal to noise ratio and, therefore, ideally a shim set under control of a computer having an automated shimming algorithm. With a second-order shim set and a shimming algorithm, a T2* $>60 \mathrm{~ms}$ at $1.5 \mathrm{~T}$ and $>30 \mathrm{~ms}$ at $4 \mathrm{~T}$ can be achieved. Since the BOLD effect is small, signal stability is extremely important. This puts very strict requirements on radiofrequency transmitter stability, phase and gain stability of the receiver, gradient driver and gradient coil stability (thermal effects in the gradient coil), shim power supply stability, and magnet field drift stability. In turn, the above listed requirements are only very small part of other requirements which should be satisfied for all the systems of a MRI device and rooms where they are located. Building vibration, magnet room temperature fluctuations, and chilled water supply temperature fluctuations, for example, can increase the instability of the final image.

\section{Conclusion}

It was considered how to construct a feature space that most accurately reflects the properties of the object under study by the addition of new measuring channels in optics, acoustics and MRI. This method has been found to improve the quality of the images obtained and promote more accurate localization of defects, but at the same time it affects data processing and hardware requirements.

The article analyzes various ways to increase the sensitivity and specificity of measurement methods in order to increase their informative value and diagnostic potential. It is shown that although the increase in sensitivity and specificity imposes new more stringent requirements for the equipment, the qualitative novelty of the information justifies all costs.

\section{References}

1. S. Tumanski, Principles of electrical measurement (CRC Press, Teylors \& Francis Group) (2006)

2. M. Hayden, P.J. Nacher, History and physical principles of MRI. Luca SABA. Magnetic Resonance Imaging Handbook, ISBN-13: 9781482216288 (2016) .www.crcpress.com...hal01191404

3. A.G. Webb (Editor), Magnetic resonance technology: hardware and system component design (2016)

4. N.K. Andreev, Methods and devices of lowfrequency relaxation NMR-introscopy (2013)

5. N.K. Andreev, NMR flowmetry and analysis in modern technologies (2004)

6. A.N. Garroway, P.K. Grannel, P. Mansfield, Image formation in NMR by selective irradiative process J. Phys, Solid State Phys, 7, 457-462 (1974)

7. N.K. Andreev, A.S. Malatsion, Dependence of NMR spin echo form on liquid flow structures Measurements 2011, Proc. of the 8th Intern. Conf. on Measurement, ISBN 978-80-969672-1-6, 221224 (2011)

8. N.K. Andreev, B.M. Odintsov, K.L. Aminov, O.I. Gnezdilov, R.M. Mubarakshin, A.A. Obynochny, R.G. Jakhin, NMR imaging using DNP and CIDNP Magnetic Resonance and Related Phenomena, Ext. Abstr. of the 27 Congress Ampere, 696 (1994)

9. N.K. Andreev, A.S. Malatsion, Sonic Testing of Long Pipes by Using Longitudinal Waves Proc, 9th Intern. Conf. on Measurement, 267-270 (2013)

10. N.K. Andreev, A.S. Malatsion, Multi-frequency acoustic method for cement bond logging based on one-sided excitation and reception of elastic waves from the top of a well, Industrial Engineering, Applications and Manufacturing (ICIEAM) 2017 International Conference Date of Conference, INSPEC Accession Number 17285072. DOI: 10.1109/ICIEAM.2017. 8076484 (2017)

11. D.R. Galeev, D.K. Zaripov, A.V. Busarev, A.B. Bel'skiı̌, I.M. Valeev, V.V. Karaev, Multichannel device for remote diagnosis of technical equipment J, Opt. Techn, 8, 486-490 (2009)

12. N.K. Andreev, A.M. Hakimov, D.Sh. Idiyatullin, New pulse sequences for T1-and T1/ T2- contrast enhancing in NMR imaging, Magn. Reson. Imaging, 16(08), 981-987 (1998)

13. N.K. Andreev, A.S. Malatsion, NMR in a flowing liquid Measurements 2011, Proc. 8th International 
Conference on Measurement (Smolenice, Slovak Republic, April 27-30, 2011), 221-224 (2011)

14. N.K. Andreev, A.S. Malatsion, NMR tomograph as an equipment for magnetic resonance detection of liquid explosives and illicit substances, Proc. MRDE-Conf. (Yalova (Istanbul): Turkey 18-23 September), 208 (2011)

15. S. Ogava, Oxigenation-sensitive contrast in magnetic resonance imaging of rodent brain at high magnetic fields, Magn. Reson. Med, 14, 68-78 (1990)

16. R.R. Price, J. Allison, R.J. Massoth, J.D. Clarke, D.J. Drost, Practical aspects of functional MRI (NMR Task Group \#8), Med. Phys, 29(8), 18921912 (2002) 